IAA-DyCoSS2 ID 37

\title{
AN EARTH-MOON SYSTEM TRAJECTORY DESIGN REFERENCE CATALOG
}

\author{
David C. Folta; Natasha Bosanac ${ }^{\dagger}$, Davide Guzzettił, and Kathleen C. Howell ${ }^{\ddagger}$
}

\begin{abstract}
As demonstrated by ongoing concept designs and the recent ARTEMIS mission, there is, currently, significant interest in exploiting three-body dynamics in the design of trajectories for both robotic and human missions within the Earth-Moon system. The concept of an interactive and 'dynamic' catalog of potential solutions in the Earth-Moon system is explored within this paper and analyzed as a framework to guide trajectory design. Characterizing and compiling periodic and quasi-periodic solutions that exist in the circular restricted three-body problem may offer faster and more efficient strategies for orbit design, while also delivering innovative mission design parameters for further examination.
\end{abstract}

\section{INTRODUCTION}

Recently-released NASA concepts for both crewed and robotic missions reveal significant interest in exploiting the Earth-Moon libration points. In fact, the dynamical structure associated with the Earth-Moon libration points may be used for a variety of applications, such as the testing and design of habitats in long-duration space missions, staging and infrastructure options for interplanetary exploration, enhancing the options for reaching lunar orbit and supporting the exploration and development of facilities on the lunar surface. ${ }^{1,2,3}$ Additional concepts for future Mars missions, asteroid rendezvous, and exploration of other solar system destinations, as published in the 2013 Global Exploration Roadmap, have also leveraged the dynamics associated with the Earth-Moon libration point orbits. ${ }^{4}$ Together, these mission concepts suggest that broader knowledge of the dynamical accessibility within the entire Earth-Moon system is warranted.

As demonstrated by ongoing concept designs and the recent Acceleration, Reconnection, Turbulence and Electrodynamics of the Moon's Interaction with the Sun (ARTEMIS) mission, there is significant interest in exploiting additional three-body dynamical structures in the design of trajectories within the Earth-Moon environment. ${ }^{5}$ Many software packages, for example, Satellite Tool Kit (STK) and NASA's General Mission Analysis Tool (GMAT), offer a graphical environment for trajectory design incorporating gravitational fields at various levels of fidelity. ${ }^{6,7}$ However, the focus of these packages is generally directed towards the delivery of trajectory designs and other actual mission support capabilities. Thus, they offer limited guidance and insight into the available dynamical structures. In recent years, the understanding of three-body dynamics within the astrodynamics community has improved tremendously, due in part to the increased utilization of techniques

\footnotetext{
* Senior Fellow, NASA Goddard Space Flight Center, Greenbelt, MD, 20771, USA.

${ }^{\dagger}$ Graduate Student, School of Aeronautics and Astronautics, Purdue University, 701 W. Stadium Ave., West Lafayette, IN, 47906, USA.

${ }^{\ddagger}$ Hsu Lo Distinguished Professor of Aeronautics and Astronautics, School of Aeronautics and Astronautics, Purdue University, 701 W. Stadium Ave., West Lafayette, IN, 47906, USA.
} 
from dynamical systems theory. As a result, there exists a wide array of known orbits with significant potential for parking, staging and transfers within the Earth-Moon system. These solutions remain the subject of much investigation to further understand the evolution of a set of orbits along any family; software such as AUTO can supply both this capability, as well as some insight into the local dynamics. ${ }^{8}$ However, no basic 'roadmap' currently exists to facilitate rapid, efficient and well-informed decisions regarding the use of these known periodic orbits for any mission prior to an end-to-end trajectory design.

In this investigation, the concept of an interactive and 'dynamic' catalog of known solutions in the Earth-Moon system is explored and analyzed as a framework to guide the design of trajectories within this dynamically sensitive environment. Specifically, such a catalog should encompass representative periodic and quasi-periodic orbits that exist in a circular restricted three-body model of the Earth-Moon system, closely approximating the true dynamical structure. Beyond low Earth orbit (LEO), the two-dimensional and three-dimensional orbit families that are currently incorporated in a 'dynamic' catalog include: libration point orbits, direct retrograde orbits about the Moon (DROs), direct prograde orbits about the Moon (DPOs), and resonant orbits. Other known families can easily be added. In fact, the capability to compute 'on-demand' by using a 'dynamic' reference, as opposed to a static database, allows for new periodic orbit families to be easily incorporated as they are discovered and better understood. In addition, a static representation of periodic solutions in the circular restricted three-body problem would be difficult to construct since the infinite orbits along each family cannot be represented analytically. Accordingly, an interactive catalog may overcome some of the challenges associated with constructing a trade space to analyze the various characteristics of a large set of solutions and their neighboring dynamics.

Periodic orbits, sampled along each family in the reference catalog, can be characterized by parameters that may aid in trajectory design and selection. Such quantities may include size, period, energy, and stability. Additional parameters of interest include station-keeping costs as well as representative maneuvers and flight times for transfers from LEO. By characterizing known periodic orbits in the Earth-Moon system and compiling this information in an interactive environment, the orbits can be filtered and compared to identify candidate solutions to potentially satisfy a given set of mission requirements. Furthermore, such a capability may offer faster and more efficient strategies for orbit design and operation, while also delivering innovative mission design parameters for further examination in tools such as the Adaptive Trajectory Design (ATD) software created at Purdue University. ${ }^{9}$ To demonstrate these capabilities, the use of a reference catalog is introduced and subsequently explored within the context of a trajectory design application for the Earth-Moon system: selection of a storage orbit for space-based infrastructure that could support lunar activities and/or enable solar system exploration.

\section{DYNAMICAL MODEL}

To facilitate rapid and intuitive exploration of the dynamical structure in the Earth-Moon system, the circular restricted three-body problem (CR3BP) is employed. This dynamical model, which serves as a reasonable approximation to the actual gravitational field, reflects the motion of a massless spacecraft under the influence of the point-mass gravitational attractions of the Earth and Moon. These two primary bodies are assumed to follow circular orbits about their mutual barycenter. The configuration of this system is depicted in Figure 1 using a coordinate frame, $\hat{x} \hat{y} \hat{z}$, that rotates with the motion of the Earth and Moon. In this frame, the spacecraft is located by the nondimensional coordinates $(x, y, z)$. By convention, quantities in the CR3BP are nondimensionalized such that the 


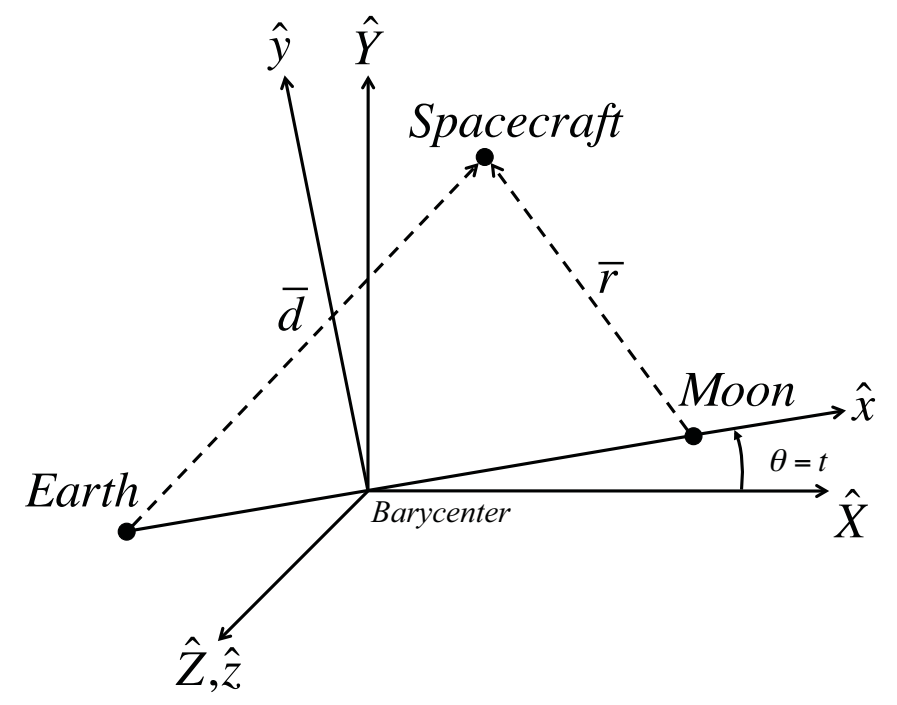

Figure 1. Definition of the rotating coordinate frame with a constant angular velocity relative to the inertial frame at a nondimensional rate of unity about the $\hat{Z}$-axis.

Earth-Moon distance is equal to a constant value of unity and their mean motion is also equal to one. In addition, the Earth and Moon have nondimensional masses equal to $1-\mu$ and $\mu$, respectively. In the rotating frame depicted in Figure 1, the equations of motion for the spacecraft can be written as:

$$
\ddot{x}-2 \dot{y}=\frac{\partial U}{\partial x}, \quad \ddot{y}+2 \dot{x}=\frac{\partial U}{\partial y}, \quad \ddot{z}=\frac{\partial U}{\partial z}
$$

where the pseudo-potential function, $U=\frac{1}{2}\left(x^{2}+y^{2}\right)+\frac{1-\mu}{d}+\frac{\mu}{r}$, where $d=\sqrt{(x+\mu)^{2}+y^{2}+z^{2}}$ and $r=\sqrt{(x-1+\mu)^{2}+y^{2}+z^{2}}$. This gravitational field admits five equilibrium points: the collinear points $L_{1}, L_{2}$ and $L_{3}$, located along the Earth-Moon line; and two equilateral points, $L_{4}$ and $L_{5}$, forming equilateral triangles with the two primaries. Since the CR3BP is autonomous, a constant energy integral exists in the rotating frame and is equal to the Jacobi Constant, $J C$ :

$$
J C=2 U-\dot{x}^{2}-\dot{y}^{2}-\dot{z}^{2}
$$

At any specific value of the Jacobi constant, there are infinite possible trajectories exhibiting a wide array of behaviors. However, any trajectory may be generally classified as one of four types of solutions: equilibrium point, periodic orbit, quasi-periodic orbit, and chaotic motion. Each of these solutions can be identified using numerical techniques and subsequently characterized using concepts and quantities from dynamical systems theory.

\section{CHARACTERIZATION OF PERIODIC ORBITS}

To construct a simple catalog to efficiently guide trajectory design, particular solutions in the form of periodic orbits are exploited. Within the framework of the CR3BP, periodic orbits exist in families and form an underlying dynamical structure. In fact, stable orbits attract trajectories in their vicinity and unstable orbits repel the nearby flow. The values of characteristic parameters, which vary continuously along the families of periodic orbits, can be analyzed to identify orbits and arcs that may be incorporated along trajectories intended to satisfy a given set of mission requirements. This concept forms the basis for the catalog that is explored within this preliminary investigation. 


\section{Orbital Parameters}

Useful characteristic quantities that represent a periodic orbit include its size, period and Jacobi constant value. To quantify the size of a periodic orbit, sample amplitudes in each of the $\hat{x}, \hat{y}$, and $\hat{z}$ directions, $A_{x}, A_{y}, A_{z}$, may supply a characterization of the size of the periodic orbit in configuration space, when viewed in a rotating frame. These amplitudes are straightforwardly calculated numerically as the absolute value of the maximum excursion of the orbit in each of the $\hat{x}, \hat{y}$, and $\hat{z}$ directions. One advantage of evaluating the 'size' of a periodic orbit is evident in the search for parking orbits that do not violate certain mission constraints. Consider, for example, line-of-sight requirements on the motion of a spacecraft with respect to either the Earth or Moon. Such a constraint on a vehicle moving along a parking orbit may be derived from limitations on the communications infrastructure. ${ }^{10}$ For a planar family of orbits, comparison of the $y$-amplitudes to the dimensions of a bounding cone may allow for preliminary identification of members that might not violate a lineof-sight constraint, prior to more computationally intensive explorations. Next, the orbital period is defined as the minimal time for the motion of a spacecraft to repeat in all the specified state variables. This parameter may be useful in the planning of maneuvers or communications operations. Finally, the Jacobi constant corresponding to each periodic orbit may be considered analogous to an energy quantity: by inspection of Eq. (2), the lower the value $J C$, the more energetic the orbit. Accordingly, comparison of the Jacobi constants along two families may provide a rough estimate of the minimum cost required to transfer between a member in each family. Such an observation is evident by maneuvers along a transfer that achieve two simultaneous results: a) a change in the velocity magnitude and, therefore, energy of the trajectory, and b) a change in the velocity direction. Thus, the transfer cost between two periodic orbits must, at the minimum, allow for the energy change, i.e., the adjustment of the Jacobi constant value. Together, these characteristic parameters may be employed to guide the design of trajectories within the Earth-Moon system.

Given that motion near a periodic orbit is influenced by the stability of the orbit, the concept of orbital stability can be used to qualitatively characterize the behavior of the nearby flow. A convenient method for evaluating stability is to analyze the eigenvalues of the state transition matrix propagated for one orbital period, i.e., the monodromy matrix. ${ }^{11}$ In the CR3BP, six eigenvalues are associated with each periodic orbit: two trivial eigenvalues equal to unity that indicate periodicity, and two reciprocal pairs of eigenvalues. ${ }^{12}$ Although computed from a local linear approximation for behavior relative to the periodic orbit, the two pairs of nontrivial eigenvalues reflect the stability of the nonlinear periodic solution. Depending on the value of each eigenvalue, in the form $\lambda=a \pm b i$, three specific cases emerge: real, complex and imaginary. Stable periodic orbits possess complex or imaginary eigenvalues on the unit circle. For each pair of complex eigenvalues, a family of quasiperiodic orbits emerges in the vicinity of the periodic orbit. Although quasi-periodic motion does not repeat over time, it traces out the surface of a torus. In combination with small maintenance maneuvers, such boundedness might be approximately retained when the quasi-periodic orbit is transitioned into a higher-fidelity gravitational environment. This behavior may be desirable for infrastructure storage or during periods of scientific observation. A pair of reciprocal eigenvalues in the form $\left|\lambda_{1}\right|=a>1$ and $\left|\lambda_{2}\right|=1 / a<1$, however, are associated with unstable periodic orbits. Such orbits possess stable and unstable manifolds which may suggest low-cost transfers to or from the orbit. Employing manifolds during trajectory design may increase the accessibility of various regions of the Earth-Moon space, or even reduce transfer costs. To simplify visualization of the eigenvalues corresponding to a periodic orbit, a stability index, $s$, is defined. This quantity is set equal to the average of the two reciprocal eigenvalues in each pair, $s=\frac{1}{2}\left(\lambda+\frac{1}{\lambda}\right)$. Stable 
orbits possess a stability index with absolute magnitude less than or equal to one, while the stability index reflecting unstable orbits is greater than one. The orbital stability index may, therefore, serve as a useful parameter for quickly and intuitively selecting periodic orbits for consideration during subsequent stages of trajectory design.

\section{Strategy to Estimate Station-Keeping Costs}

To assess and compare the maneuver costs to maintain selected periodic orbits, a long-term station-keeping strategy is employed. ${ }^{10}$ A variety of options for station-keeping are possible; one specific approach is currently implemented. First, a reference solution is defined, one that is comprised of twelve revolutions of a periodic orbit. For each orbit, station-keeping maneuver locations are also identified. As an example, for the DRO family, a maneuver location is selected as the $x$ axis crossing. Next, a random error is applied to the initial state along the reference solution. In this investigation, position errors are assumed with a gaussian distribution described by a mean of $0 \mathrm{~km}$ and a standard deviation of $1 \mathrm{~km}$ in each direction, while velocity errors have a distribution centered around $0 \mathrm{~cm} / \mathrm{s}$ and a standard deviation of $1 \mathrm{~cm} / \mathrm{s}$. The disturbed initial state is integrated forward in time in the CR3BP until the next station-keeping maneuver location, with each maneuver computed using the remaining portion of the reference solution as an initial estimate. Constrained optimization enforces continuity along the reference path and specifies that the endpoint along the reference path occurs at the same position as the initial point. Each maneuver is also subject to a maneuver execution error randomly assigned using a gaussian distribution with a standard deviation of $1 \%$. This new state is then integrated forward to the subsequent station-keeping maneuver location. The entire process is repeated until the end of the reference or baseline trajectory. An example of the maintenance of an $L_{1}$ halo orbit is displayed in Figure 2, demonstrating the success of the long term station-keeping strategy. For each simulation where the optimization process converges, the total station-keeping $\Delta V$ is calculated as the sum of the magnitudes of each maneuver. For each orbit, the algorithm completes 500 trials and the average of the total $\Delta V$ is extrapolated to produce a representative estimate of the cost required to maintain a selected periodic orbit for one year. Given onboard propellant limitations for a spacecraft, a long-term station-keeping estimate can be used in preliminary analyses to select a candidate periodic orbit prior to higher-fidelity modeling and more precise maneuver computations. Other station-keeping strategies can also be incorporated.

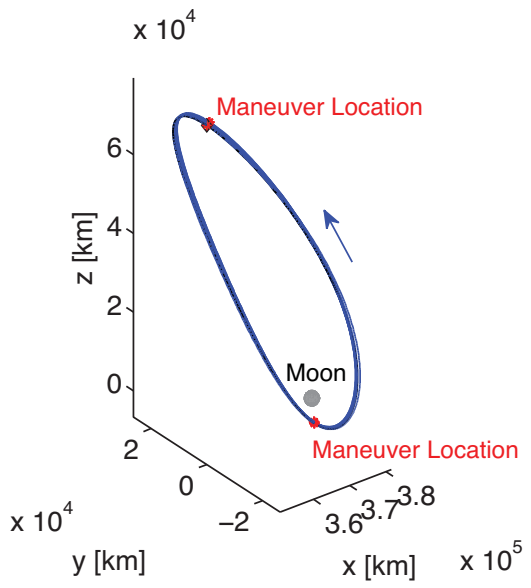

Figure 2. Maintenance of an $L_{1}$ halo orbit via long term station-keeping. 


\section{Strategy to Estimate Transfer Costs from Low Earth Orbit}

The viability of reaching each periodic orbit from LEO can be measured using some representative transfer cost and time-of-flight (TOF). For preliminary exploration of the use of a reference catalog in trajectory design, this investigation incorporates only direct transfers involving two impulsive burns: one $\Delta V$ to depart a $300 \mathrm{~km}$ altitude LEO, i.e., $\Delta V_{L E O}$, and one $\Delta V$ to insert onto the desired periodic orbit, $\Delta V_{P O I}$. Of course, multiple-burn transfers, lunar flybys and manifold connections could reduce the transfer and insertion costs; more extensive options and various approaches to construct transfers could be incorporated into more complex implementations of a reference catalog. In any case, the goal here is a set of simplified metrics to trade-off various scenarios and allow new concepts to emerge. Thus, the initial estimate for each direct transfer is constructed using a conic arc with a 300-km altitude periapsis and an apoapsis radius equal to the distance from the Earth to a selected point along the periodic orbit. This estimate is then corrected within the CR3BP using a multiple shooting algorithm to ensure continuity at all interior points. At the initial point, the transfer is constrained to ensure that it links to the LEO in position only, with one additional constraint that the departure maneuver, $\Delta V_{L E O}$, is tangential to the velocity vector. ${ }^{13}$ The terminal point of the transfer arc must also possess the same position vector as a prescribed location along the orbit (within the specified tolerance). Once corrected, the transfer is optimized using a sequential quadratic programming algorithm in MATLAB's fmincon function, subject to the described constraints. The objective function for the optimization process is set equal to the magnitude of $\Delta V_{P O I}$, a quantity that is most indicative of the cost associated with reaching a desired periodic orbit. ${ }^{13}$ Furthermore, the target location along a given periodic orbit is selected by constructing a family of transfers along one member of each family and observing the location at which $\triangle V_{P O I}$ is a minimum; such a location could, for example, be the periapsis, an $x$-axis crossing, or the location of maximum $z$-excursion. Using this methodology, transfers can be constructed from LEO to any periodic orbit in the CR3BP and the 'costs' can be reasonably compared.

One assumption in computing transfers to periodic orbits involves the inclination of the LEO relative to the $x y$-plane in the CR3BP. In an ephemeris model of the Earth and Moon, the inclination of the Moon is observed to oscillate between $18.14^{\circ}$ and $28.72^{\circ}$ relative to the Earth's equator, with a mean inclination of $23.5^{\circ}$. In addition, the $x y$-plane in the CR3BP is defined as the instantaneous plane of the Moon's orbit. Therefore, the inclination of a $28.5^{\circ} \mathrm{LEO}$ with respect to the $x y$-plane in the CR3BP also varies with the same amplitude and a mean value of $4.99^{\circ}$. Accordingly, the variation in the lunar inclination is incorporated into this investigation by computing transfers to a three-dimensional periodic orbit from a LEO with three different inclinations relative to the $x y$ plane in the CR3BP: $i_{\text {min }}=2^{\circ}, i_{\text {mean }}=4.99^{\circ}, i_{\max }=10.37^{\circ}$. Note that these three inclinations do not encompass the entire range of LEO inclinations relative to the $x y$-plane in the CR3BP, but are straightforward to implement and offer reasonable comparisons. For transfers to planar periodic orbits, however, the LEO and the transfer arc are assumed to lie within the $x y$-plane, for simplicity. Together, these inclination assumptions allow for representative direct transfers to be computed and employed throughout the investigation.

As an example of the process, transfers are constructed to sample members of the DRO family. First, consider a transfer to a planar DRO with a period of 20.5 days, as depicted in Figure 3(a). In this figure, the Earth and Moon are represented by gray dots, with $L_{1}$ and $L_{2}$ indicated by green diamonds. The target DRO orbit is colored red, while the planar transfer arc is black, with arrows indicating the direction of motion. As evident in the figure, the transfer location along the DRO is selected as the furthest $x$-axis crossing from the Earth; this location is close to a minimum 


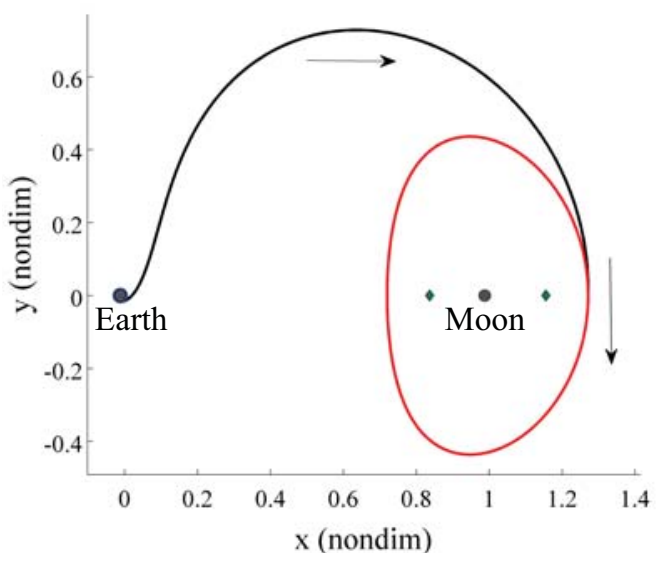

(a) Transfer to planar DRO with a period of 20.5 days.

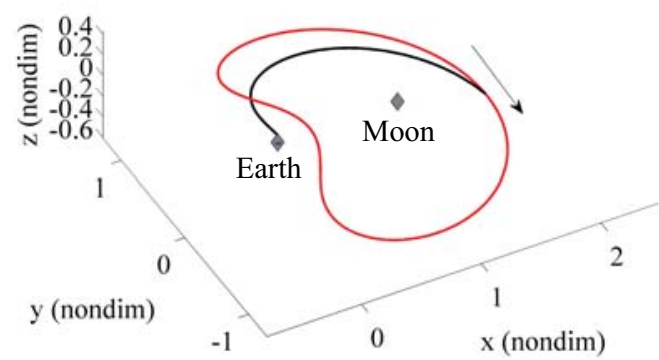

(b) Transfer to 3D DRO with a period of 27.1 days.

Figure 3. Transfer examples.

in the required $\Delta V_{P O I}$ for insertion. The final transfer trajectory includes a maneuver, $\Delta V_{L E O}$, equal to $3.12 \mathrm{~km} / \mathrm{s}$, a periodic orbit insertion maneuver, $\Delta V_{P O I}$ equal to $581 \mathrm{~m} / \mathrm{s}$ and a flight time corresponding to 7.06 days. A similar transfer is constructed for a three-dimensional DRO with a much larger period of 27.1 days, from a LEO with inclination $i_{\text {mean }}=4.99^{\circ}$. This sample transfer is depicted in Figure 3(b), with the same color scheme as in Figure 3(a). For this example, the transfer requires a $\Delta V_{L E O}$ value of $3.48 \mathrm{~km} / \mathrm{s}$, a periodic orbit insertion maneuver, $\Delta V_{P O I}$, equal to $423 \mathrm{~m} / \mathrm{s}$ and a flight time of 8.23 days. Both transfers are then continued along their corresponding family using natural parameter continuation, yielding a measure of the comparative cost required to reach each member of the family; such a process is extended for comparison across families as well.

\section{CLASSIFICATION OF PERIODIC ORBIT FAMILIES}

Although an infinite number of families of periodic orbits exist in various regions of the EarthMoon space as modeled in the CR3BP, the most familiar periodic orbits in this regime are associated with the libration points. Lying in the Earth-Moon plane, Lyapunov orbits emanate from each of the collinear libration points. Examination of the center manifold associated with each collinear point in the Earth-Moon system also yields a family of vertical orbits, which extend out of the plane. Other three-dimensional libration point orbits include northern and southern halo families, axials, and butterfly orbits. Sample members of these families are depicted in Figures 4(a)-4(c). Several of these families of periodic orbits have been explored for potential mission concepts involving, for instance, staging and infrastructure placement for solar system exploration missions or even scientific observation. ${ }^{4}$ One mission has already succeeded in demonstrating the utility of trajectories that exploit three-body dynamical structures in the Earth-Moon system: ARTEMIS. ${ }^{5}$ The pair of spacecraft, with a focus on examining energetic particle accelerations near the Moon, leveraged manifold connections between $L_{1}$ and $L_{2}$ quasi-halo orbits, allowing an extended mission despite relatively small propellant availability. In addition, planar short- and long-period orbits also exist in the vicinity of the equilateral libration points, $L_{4}$ and $L_{5}$. Out-of-plane vertical and axial families are also nearby, as plotted in Figure 4(d). Periodic motions in the vicinity of the equilateral libration points have recently been considered for the asteroid retrieval mission, ${ }^{14}$ and briefly utilized during the Hiten mission. ${ }^{15}$ Due to these potential mission applications, periodic orbits in the vicinity of each of the equilibrium points in the Earth-Moon system are currently the subject of much interest. 


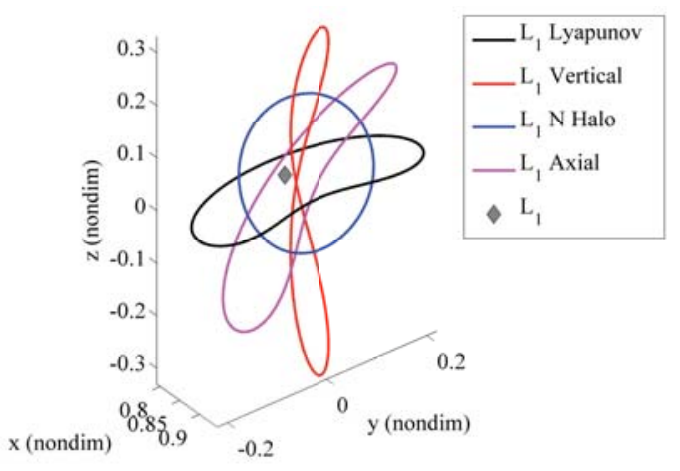

(a) $L_{1}$ Orbit Families

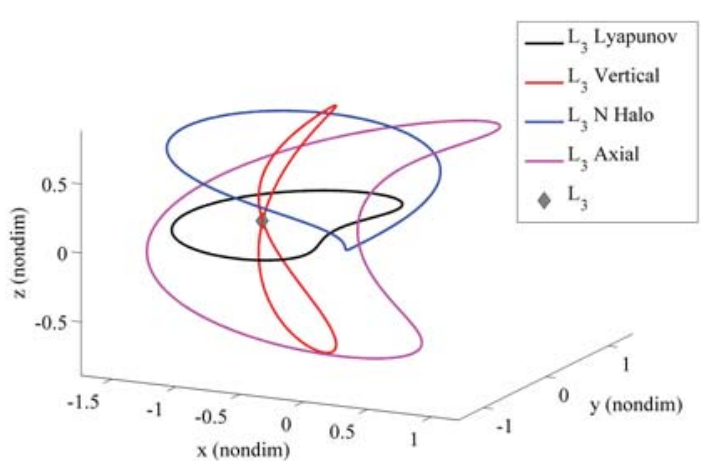

(c) $L_{3}$ Orbit Families

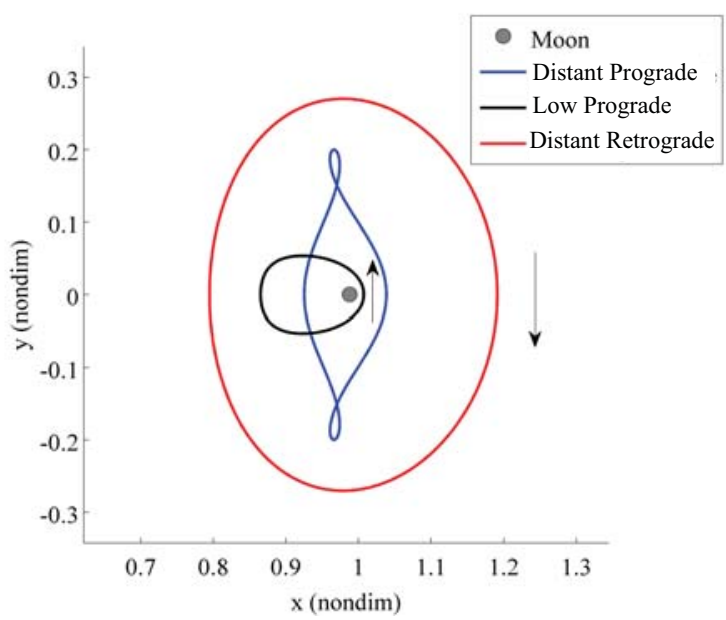

(e) Moon-Centered Orbit Families

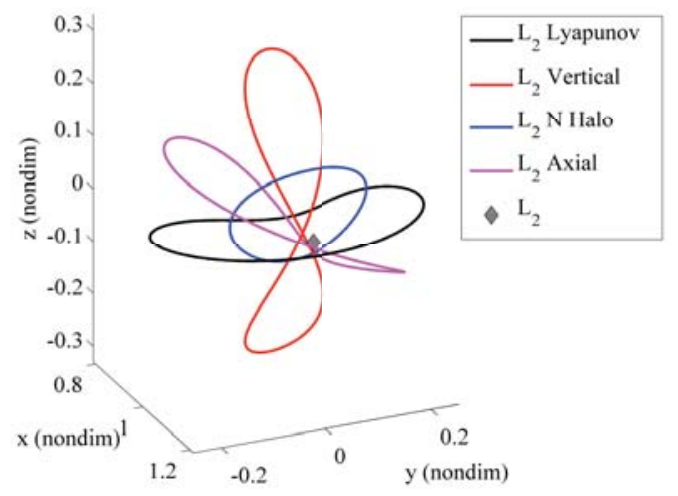

(b) $L_{2}$ Orbit Families

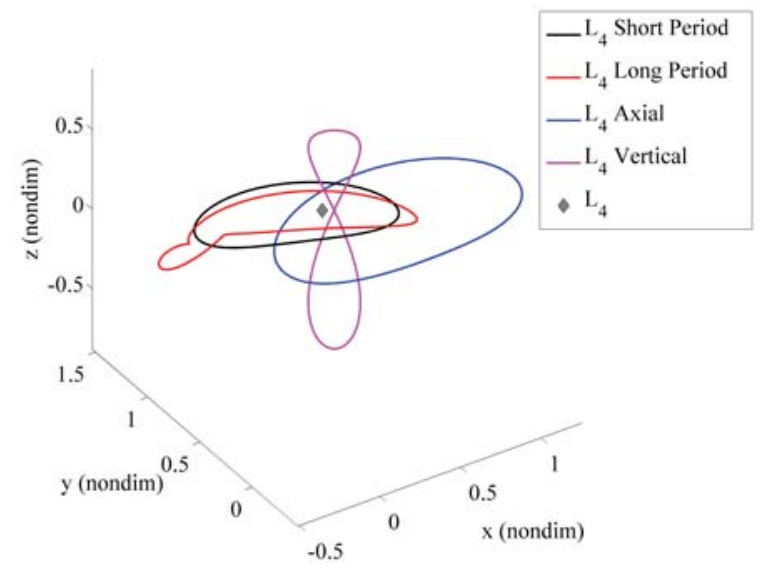

(d) $L_{4}$ Orbit Families

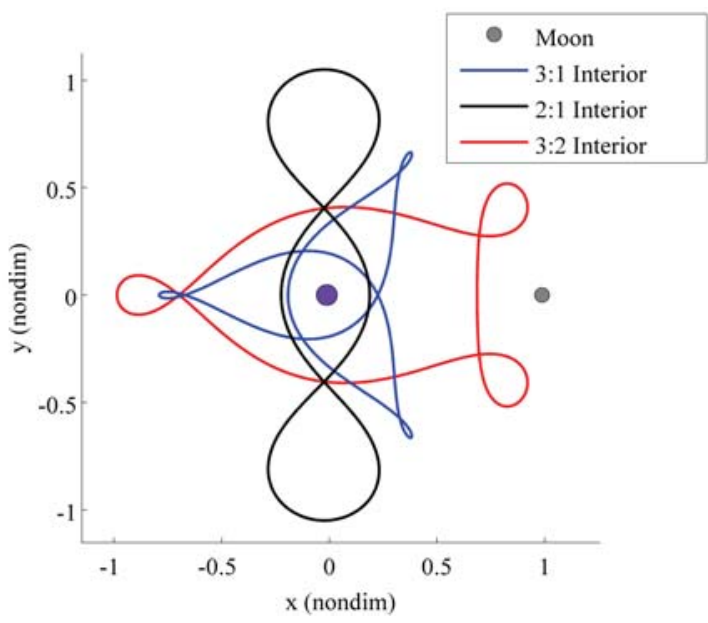

(f) Sample Resonant Orbit Families

Figure 4. Sample members of well-known orbit families in the Earth-Moon system, plotted in the rotating frame. 
Another type of periodic orbit family that is potentially useful in the Earth-Moon system is the set of resonant orbits, both planar and three-dimensional. Resonant orbits, pictured in Figure 4(f), possess orbital periods that can be approximately described as an integer ratio between the orbital period of the spacecraft and the period of the Moon's orbit. Although resonant families are commonly understood within the context of solar system dynamics, they are not widely employed in trajectory design within the Earth-Moon system. One prominent example of the utility of resonant orbits in Earth-Moon trajectory design, however, is the Interstellar Boundary Explorer (IBEX) mission; the IBEX spacecraft was launched in 2008. ${ }^{16}$ In the extended mission, IBEX successfully exploited a 3:1 resonance that possessed favorable stability properties. Another example involves a 2:1 resonant orbit about the Earth, intended for use during a scientific observation phase of the upcoming Transiting Exoplanet Survey Satellite (TESS) mission. ${ }^{17}$ There is, therefore, merit in considering the contribution of resonant families to the underlying dynamical structure in the EarthMoon system and any potential impact from leveraging these relationships.

Additional families of interest include periodic orbits that encircle either the Earth or the Moon. In particular, consider three Moon-centered families of orbits, depicted in Figure 4(e): direct retrograde orbits, distant prograde orbits and low prograde orbits. The DRO family is predominantly comprised of stable members that travel clockwise around the Moon when viewed in a rotating frame, as depicted in Figure 4(e). Since a stable orbit can potentially require little propellant for maintenance, DROs have been considered as viable candidates for long-term storage orbits in asteroid retrieval mission concepts. ${ }^{18}$ The distant and low prograde orbits display, in general, counterclockwise motion about the Moon, and their families possess some resonant members. Recently, the DPOs have been examined as transfer mechanisms between $L_{1}$ and $L_{2}$ Lyapunov orbits, and as lunar parking orbits. ${ }^{919}$ Each of these families also possesses three-dimensional counterparts that can influence the dynamical behavior in the vicinity of the Moon.

Families of periodic orbits that are currently familiar to the astrodynamics community can be categorized to facilitate construction of a reference catalog. In this investigation, four classes of periodic orbits are defined: libration point orbits, resonant orbits, Earth-centered orbits and Mooncentered orbits. The periodic orbits considered in this preliminary reference catalog are organized into these families, as displayed in Figure 5. For clarity, families in each of the classes considered in this investigation are colored green, red or blue. Note that the only type of Earth-centered orbit utilized thus far in this investigation is LEO. Accordingly, families that are categorized as Earthcentered orbits are colored grey. In addition, the boxes corresponding to each orbit family can encompass both two-dimensional and three-dimensional members. Although all members of any periodic orbit family may not belong exclusively to one class or one family, as indicated by the black arrows, an intuitive categorization may aid in the application of an interactive reference database to search for a desired solution without precise knowledge of its name, shape or evolution. Such a capability is especially important for users not familiar with the intricacies of the CR3BP and the connections between various types of orbits and their respective families.

\section{Composite Representations of Periodic Orbit Families}

To simultaneously compare a large number of orbit families, a simple composite representation is constructed. Since the evolution of orbital parameters along a family can reflect a complex and nonlinear relationship, it is challenging to simultaneously visualize several characteristic curves. Accordingly, a simple statistical summary, representative of each family, is developed in the form of its mean, range and standard deviation; such measures facilitate the construction of an aggregate 


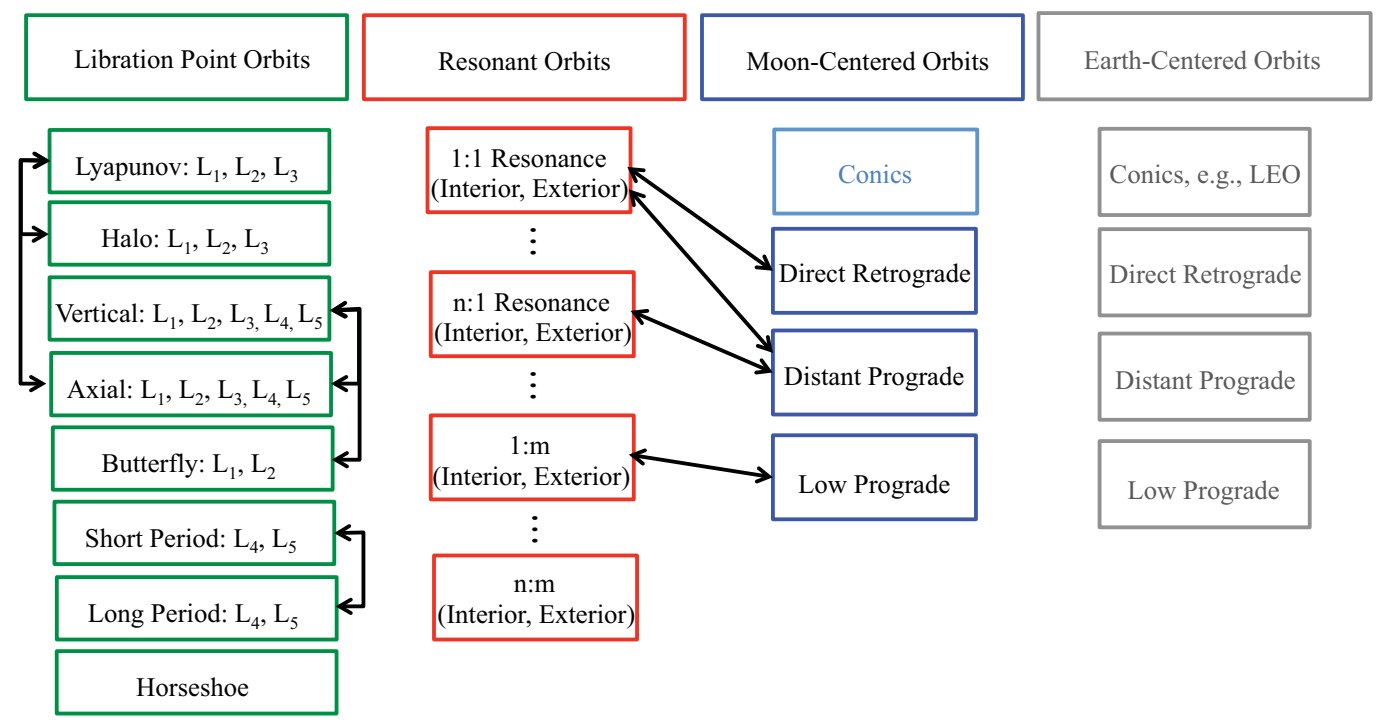

Figure 5. Organization of orbit families included in reference catalog to date, with selected links indicated; the framework is easily expanded as new options emerge.

representation of a large set of families. Consider, for example, Figure 6(a), which approximately summarizes an average stability reflected by the various orbits in each family; such a representation is currently available in the reference catalog, with each family identified by the abbreviations in the legend in Figure 6(b). Each family is represented by a single circle located at the mean value of the stability index computed for each orbit along the family. For each circle in Figure 6(a), the radius is equal to a scaled value of the range of the parameter values across the family. Although such a plot does not precisely represent the range in the stability index encompassed by the orbits across a complete family, it can be employed for preliminary identification of candidate families for further examination. As an example, families represented by small circles located near a value of the stability index equal to unity may indicate that a family is comprised of a significant range of stable members that are likely surrounded by bounded quasi-periodic motion, such as the family of planar DROs. Alternatively, a small circle located at a relatively large value of the mean stability index may indicate that the family is dominated by unstable periodic orbits. Such an observation may be useful during the search for unstable periodic orbits that may offer a transfer mechanism to access various regions of the Earth-Moon space.

To visualize the range of a certain quantity across all families for comparison, a composite representation can be constructed using bars. Consider, for example, the search for candidate orbit families that may be connected by low-cost transfers. Two periodic orbits at comparable energy levels may, in fact, possess these low-cost links. To guide this search, Figure 7 illustrates a bar representation of the Jacobi constant, an energy-like quantity, for all families now available in the reference catalog. Recall that a legend of abbreviations is displayed in Figure 6(b). Each bar encompasses the range over the Jacobi constant values corresponding to various orbits along a family, with the lower and upper bounds indicated by horizontal lines. Any bars that overlap vertically indicate that some members from each family exist at similar energy levels. To demonstrate the use of the plot in Figure 7, consider a transfer to a DPO. Since the Jacobi constant values along the DPO family exist at much higher values than the Jacobi constant across the three-dimensional DRO family, it is unlikely that low-cost connections exist between any members of these two families. In 

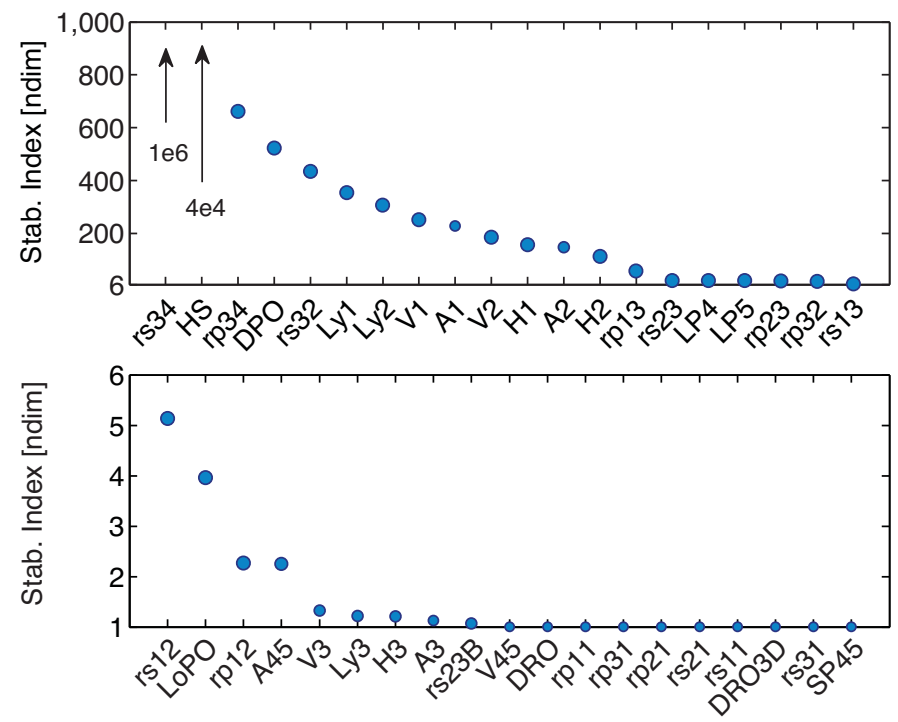

(a) Composite dot representation of the stability index across all families.

$$
\begin{array}{lcc}
\text { Lyi }=\text { Lyapunov for } \mathrm{L}_{\mathrm{i}} & \mathrm{rpnm}=\text { Planar Resonant } & \mathrm{DRO}=\text { Planar Distant } \\
\mathrm{Hi}=\text { Halo for } \mathrm{L}_{\mathrm{i}} & \mathrm{n}: \mathrm{m} & \text { Retrograde } \\
\mathrm{Ai}=\text { Axial for } \mathrm{L}_{\mathrm{i}} & \mathrm{rsnm}=\text { Spatial Resonant } & \text { DRO3D }=\text { Spatial } \\
\mathrm{Vi}=\text { Vertical for } \mathrm{L}_{\mathrm{i}} & \mathrm{n}: \mathrm{m} & \text { Distant Retrograde } \\
\mathrm{SPi}=\text { Short Period for } \mathrm{L}_{\mathrm{i}} & & \mathrm{DPO}=\text { Distant Prograde } \\
\mathrm{LPi}=\text { Long Period for } \mathrm{L}_{\mathrm{i}} & & \mathrm{LOPO}=\text { Low Prograde } \\
\mathrm{HS}=\text { Horseshoe } & &
\end{array}
$$

(b) Legend of abbreviations, colored by category.

Figure 6. Sample dot representation of characteristic quantities of all families available in the reference catalog.

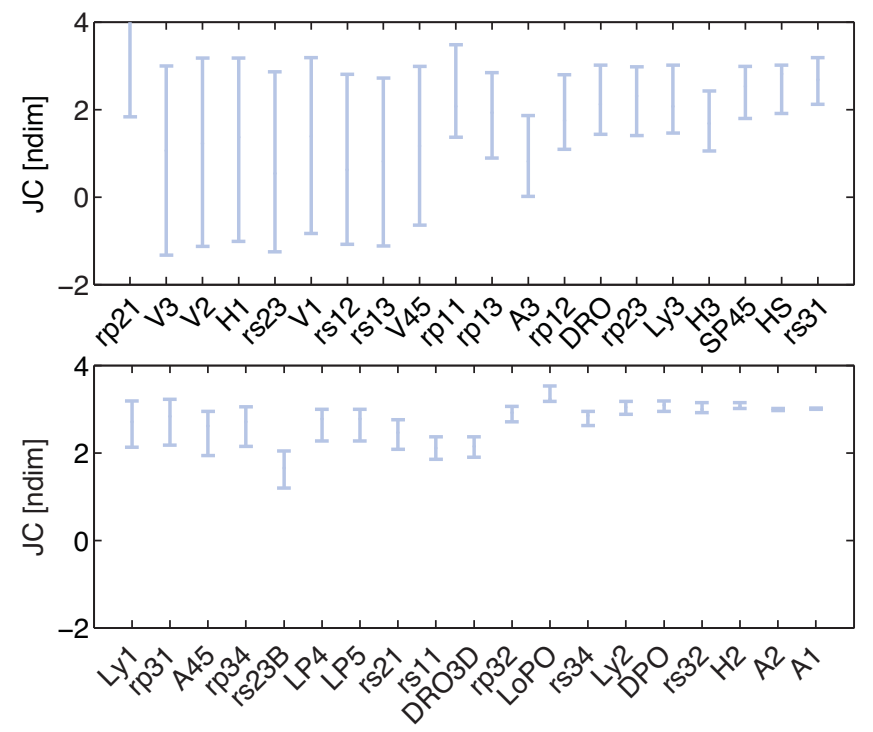

Figure 7. Bar representation of the Jacobi constant of all families available in the reference catalog. 
contrast, the ranges of the Jacobi constant values over the $L_{1}$ Lyapunov family of orbits in comparison to the DPO family do overlap. Accordingly, a more extensive numerical search for low-cost links between some members of the two families would be warranted; in fact, low-cost transfers do exist between these two families. ${ }^{9}$ This capability for rapid and efficient identification of candidate orbits satisfying a particular mission objective may be useful during the trajectory design process.

\section{CONCEPTUAL OVERVIEW OF AN INTERACTIVE REFERENCE CATALOG}

A conceptual overview of an interactive reference catalog that offers intuitive access to the dynamical structures available in the Earth-Moon system is now demonstrated. In particular, this concept is developed to allow an iterative examination of a large set of well-known periodic orbit families. It is assumed that a critical capability is the selection of a range of orbits that can be stored and exported for later examination in a trajectory environment such as ATD. ${ }^{9}$ A conceptual overview of the reference catalog concept is summarized and displayed in Figure 8, with two paths available for orbit selection.

A first potential strategy for orbit selection involves the analysis of individual families in the catalog, categorized as depicted in Figure 5. In the top left of the conceptual overview in Figure 8, one orbit family that warrants consideration may originate from any of the four categories and it is useful to view a summary of the parameters describing members along a selected family. Filters can also be applied to narrow the range of values for the characteristics quantities along each family size, period, Jacobi constant and stability index - and plot the remaining members from the selected family in configuration space. Since this process is inherently iterative, it may be beneficial at any time to select additional families and add them to the plot with individually defined filters. To represent one of the characteristic quantities along each family, a color scale can also be introduced. Once all of the desired filters have been applied, the remaining periodic orbits, belonging to any number of families, are input to a workspace.

An alternative strategy for orbit selection originates with a more global portrait of the periodic orbit families available in the catalog. Beginning at the bottom left of Figure 8, a designer may visualize a statistical summary of one characteristic quantity for every family in the catalog. This visualization is realized using plots such as the dot and bar representations portrayed in Figures 6 and 7. As an example of the interactive nature of such a reference catalog, consider the flexibility achieved by a switch, at any time, between the two types of representations or an option to modify the characteristic quantity on the vertical axis. By point-and-click selection, candidate families may be selected for closer examination and comparison. During this next stage of the orbit selection process, the candidate families may be represented in a two-parameter space. At this time, the parameters to be selected on either the vertical or horizontal axes include size, period, Jacobi constant, stability index, transfer cost, transfer TOF, and station-keeping cost. At any time, the characteristic quantities on each axis can be modified. With further insight into the characteristic quantities along each family, ranges along families of periodic orbits may be selected and input to a workspace.

Once suitable sets of periodic orbits are identified, the selections can be viewed in the catalog or reference workspace. In this final step, the desired periodic orbits are plotted in configuration space and their characteristic quantities summarized in a table. A range of periodic orbits is then selected to export to a data file or for use in a more complete trajectory design environment such as ATD. ${ }^{9}$ For demonstration of the process, an illustrative example is useful. Thus, the interactive reference database concept is now applied to the search for a possible storage orbit for space-based infrastructure that may be support lunar or solar system exploration. 

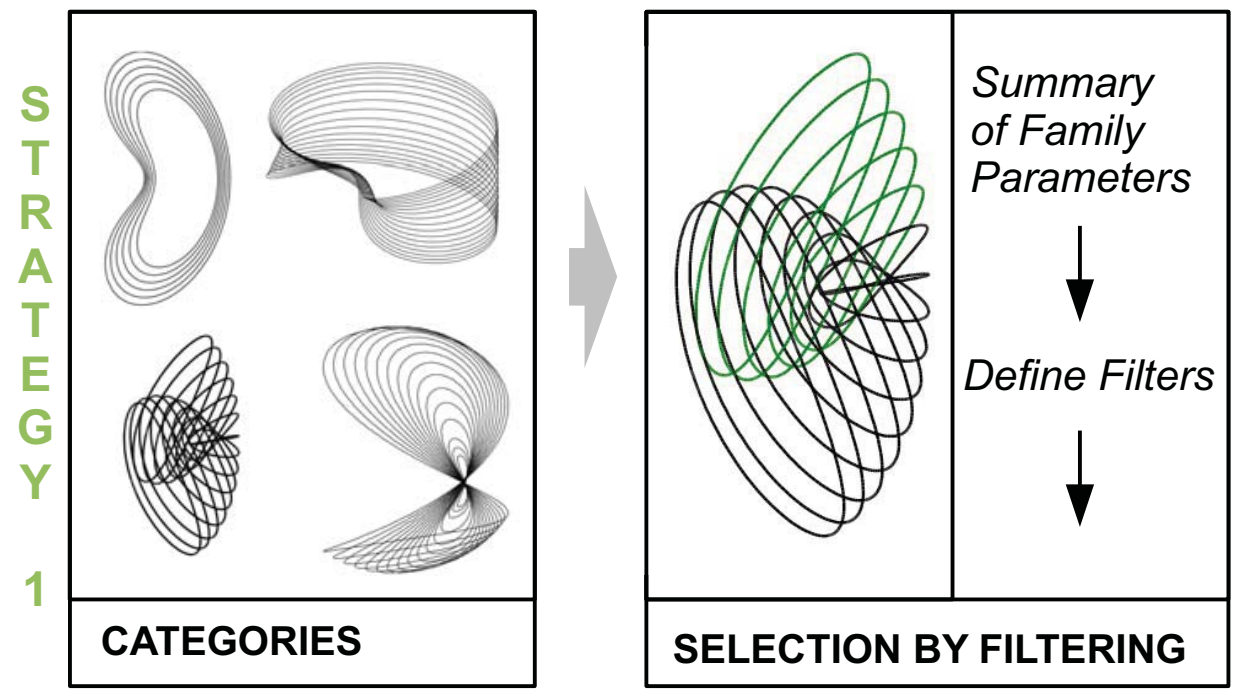

Selected ranges along various families
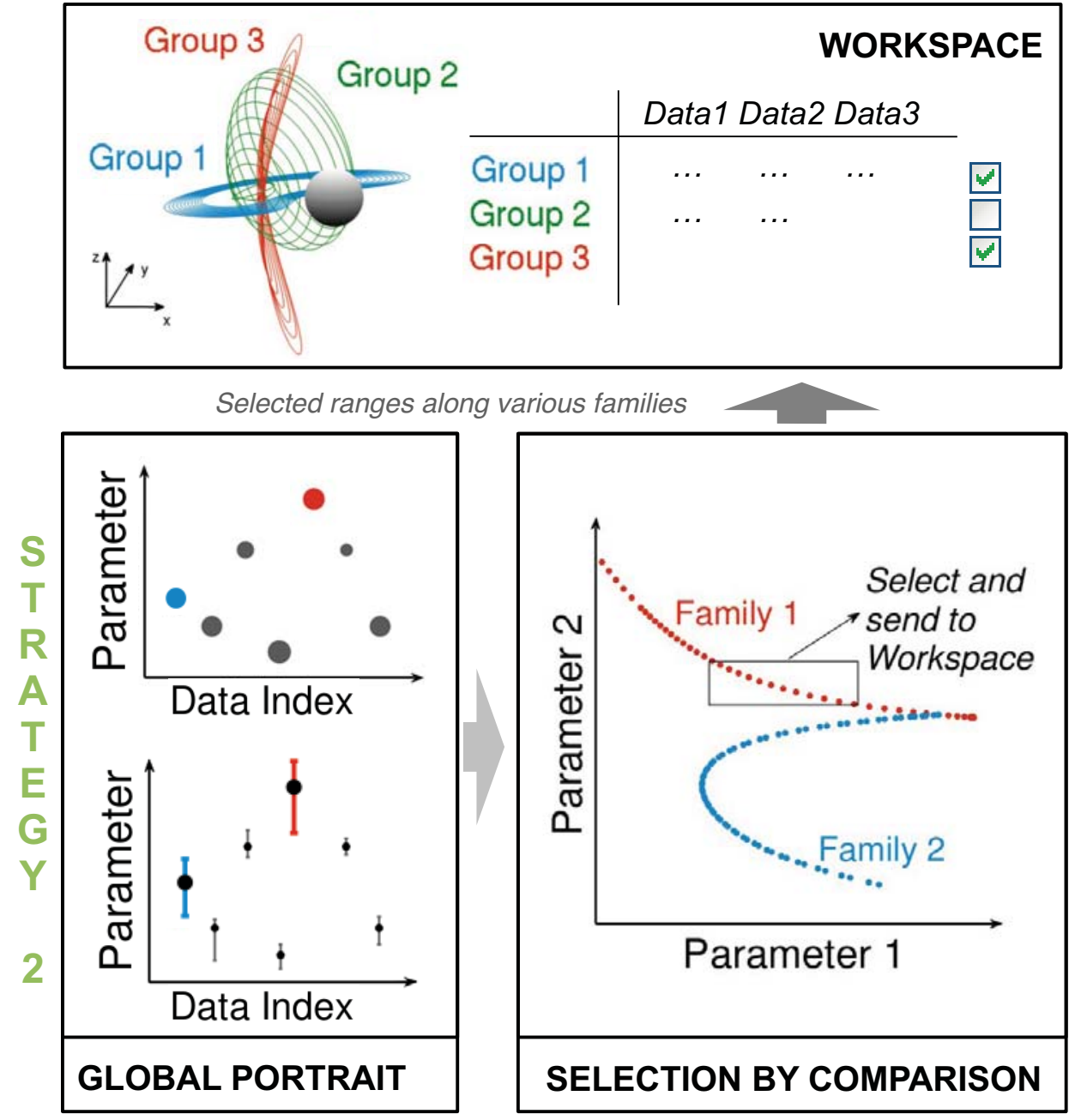

Figure 8. Graphical representation of the reference catalog concept. 


\section{APPLICATION OF REFERENCE CATALOG TO TRAJECTORY DESIGN IN THE EARTH- MOON SYSTEM}

To demonstrate the utility of this interactive catalog approach, consider a recent mission concept of interest: storage options for infrastructures that may facilitate lunar activities or further exploration of the Moon or the solar system. Such infrastructures could, for example, exist in the form of a propellant depot, or an experimental long-duration habitat. For this sample mission, a set of requirements are assumed. First, the orbit is to be maintained for a relatively long time interval. Since the $\Delta V$ required to reach Mars, for example, is lowest once every synodic period, 780 days, it may be desirable for a propellant depot to remain in the selected storage orbit for at least several years. A similar conclusion drives the assumption for the storage orbit of an experimental long-duration habitat. This requirement that the storage orbit be maintained for several years is translated into a requirement that the station-keeping cost should be reasonably small. In addition, it is desirable that the storage orbit be easily accessible from the $L_{1}$ and $L_{2}$ Lyapunov orbits, which may be employed for staging options. Alternatively, the manifolds of the Lyapunov orbits might possibly be exploited as transfer mechanisms to various asteroids and Mars. ${ }^{20}$ This requirement suggests that the storage orbit should possess a Jacobi constant comparable to that of the $L_{1}$ and $L_{2}$ Lyapunov families. In addition to these two requirements, bounds on other characteristic quantities are employed based on intuition to constrain the acceptable range of values. For example, a candidate orbit could be sought that does not possess a large stability index, thereby limiting the departure options in terms of exploiting the local dynamics in the vicinity of the reference periodic solution. A 'fuzzy' constraint may also be introduced on the period and geometry of any candidate orbits to avoid strong limitations on the launch opportunities for on-orbit rendezvous. Candidates for a storage orbit satisfying these constraints may be selected using the interactive reference catalog.

\section{Selection of Candidate Orbits}

To identify candidate storage orbits, a global portrait of the families available in the 'dynamic' catalog may be the most appropriate entry point. First, the composite bar representation displayed in Figure 7 is utilized. Recall that the candidate storage orbits should include members with Jacobi constant values that are comparable to the $L_{1}$ and $L_{2}$ Lyapunov families. From this figure, it is clear that the $L_{3}$ axial family and the Moon-centered low prograde family do not meet this loose constraint. Accordingly, these families likely do not possess members that are easily accessible to or from either an $L_{1}$ or $L_{2}$ Lyapunov orbit. The three-dimensional DRO family can also be excluded, since their range of Jacobi constant values do not exist close to the values in the $L_{2}$ Lyapunov family. Families of orbits that are not viable are, therefore, purposefully deselected.

Next, constraints on the period and geometry of any candidate orbit are applied using, respectively, a composite bar representation for the period as well as plots of selected members across a family in configuration space. As displayed in Figure 9, the $L_{4}$ and $L_{5}$ long-period orbits possess a large mean period of approximately 107 days. Since the corresponding range for these families is small, all members clearly possess a relatively large period. A similar conclusion is quickly apparent for the horseshoe orbits and many of the resonant families. Since their periods are large, rendezvous opportunities to these orbits may be difficult. Accordingly, these families can be immediately discarded in the search for a candidate storage orbit. In addition, the $L_{3}$ Lyapunov, halo, axial and vertical families are located far from the $L_{1}$ and $L_{2}$ Lyapunov orbits and the Moon, when viewed in a rotating frame. This relative geometry may limit the accessibility and utilization of any infrastructure in an $L_{3}$-centered storage orbit. Furthermore, the 3:1 and 2:1 resonant families do not 

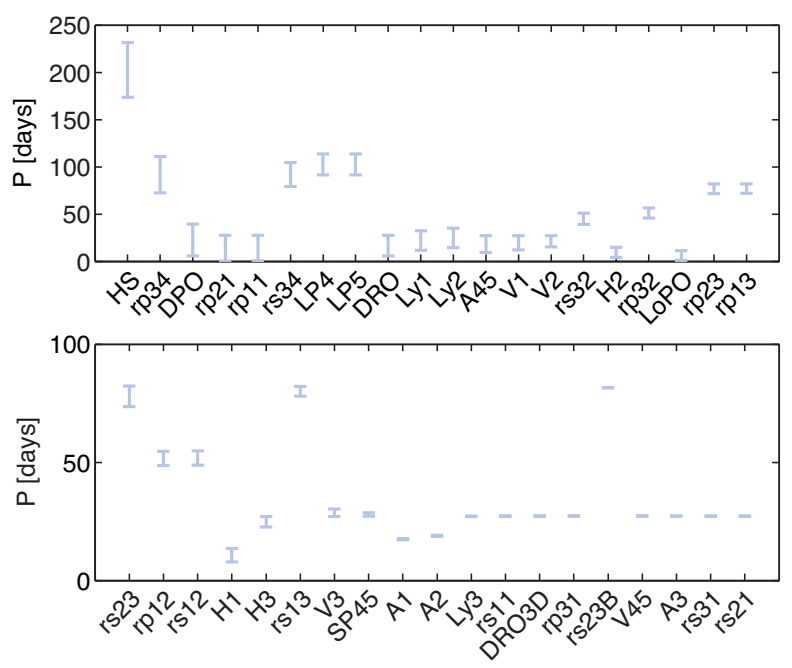

Figure 9. Composite representation of period of the orbits in the families available in the reference catalog.

exhibit a close lunar passage and may not sufficiently support lunar activities. These families can, therefore, be excluded from the set of candidate orbits. The only remaining resonant orbit in the set of candidates is the spatial 1:1 resonant family, which is simply a three-dimensional DRO. This family has already been excluded via a Jacobi constant filter.

The remaining families of periodic orbits can be further analyzed, with candidates selected through a comparison of the transfer and station-keeping costs. First, a simple box representation of the transfer and station-keeping costs, such as that in Figure 10, can be constructed using information from the interactive catalog. Analogous to the dot plot, the center of each box is located horizontally at the mean annual stationkeeping $\Delta V$ value and vertically at the mean $\Delta V_{P O I}$. Each box is sized using the standard deviation in the corresponding direction. Note that the representative station-keeping cost corresponding to each family is dependent upon the number of Monte Carlo trials and the size of the errors applied to each state and maneuver, whereas the cost of a direct transfer to a specific location along each orbit is not a statistical quantity. Although Figure 10 does not precisely reflect all the station-keeping and transfer results throughout each complete family, the figure enables a simple visualization in contrast to a more complex (and more precise) representation as plotted in Figure 11. Using either Figure 10 or 11 as a reference, however, a number of observations offer insight into the suitability of each candidate family. First, the $L_{1}$ Lyapunov and $L_{2}$ halo families may require a large $\Delta V_{P O I}$ for a transfer from LEO, assuming the simple direct transfer concept without any assistance in the form of a lunar flyby. On average, the DRO and $L_{1}$ halo families require the least $\Delta V_{P O I}$. Similarly, the $L_{1}$ and $L_{2}$ halo families possess many members that are expensive to maintain via the sample long-term station-keeping strategy employed in this investigation. On average, periodic orbits from the DRO family appear to require relatively little in terms of annual station-keeping $\Delta V$. Although there are families in the vicinity of $L_{4}$ and $L_{5}$ that require both low transfer and station-keeping costs, these orbits do not remain sufficiently close to the Moon at all times. While a number of the other orbit families included in these transfer diagrams may possess potential candidates for further analysis, the DRO family seems most interesting due to its favorable geometry, low station-keeping costs over nearly the entire range of orbits and low periodic orbit insertion $\Delta V \mathrm{~s}$ assuming a straightforward transfer from LEO. 


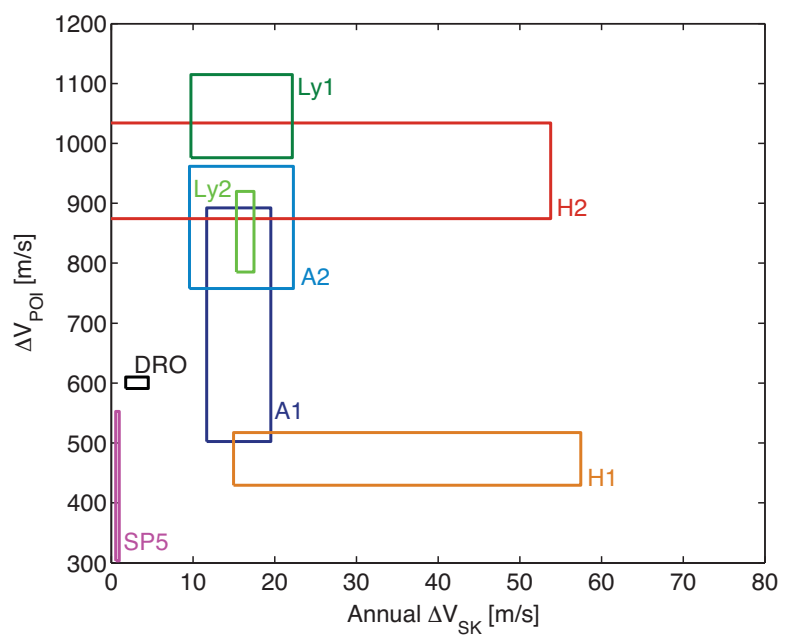

Figure 10. Simplified representation of transfer and station-keeping cost of each family using boxes.

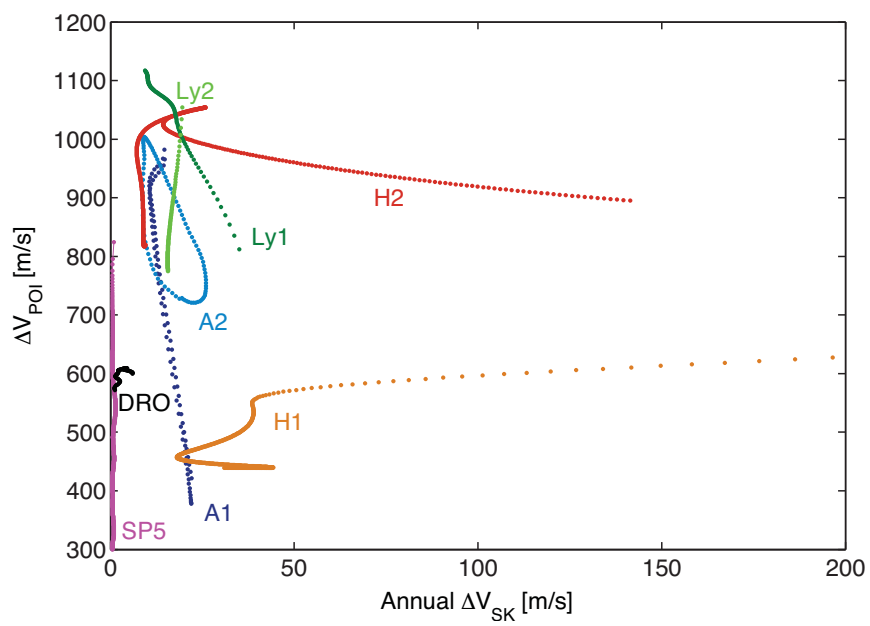

Figure 11. Characteristic curves of transfer and station-keeping cost of each family.

\section{Analysis of DRO Family}

Given that most members of the DRO family appear to meet the previously defined requirements for a storage orbit that supports a space-based infrastructure, the family can be analyzed further in a catalog workspace. First, the DROs are isolated on a transfer diagram, as depicted in Figure 12. On the vertical axis in each plot is the insertion cost, $\Delta V_{P O I}$, to reach a DRO from a LEO that is assumed to lie within the $x y$-plane, while the horizontal axis corresponds to the annual station-keeping $\Delta V$. Both quantities are displayed as $\mathrm{m} / \mathrm{s}$. In Figure 12(a), the one parameter curve representing the DRO family is colored by the TOF associated with the transfer from LEO. Since an interactive environment allows modification of the visualization parameters at any time, Figure 12(b) displays the same curve colored by the period of each orbit in the DRO family. Using these two figures as a reference, observe that the period of the DROs monotonically increases as the family evolves away from the Moon. The transfer TOFs from LEO to the DROs appear to follow a similar pattern. From these observations, it is apparent that larger DROs with periods greater than 16 days 


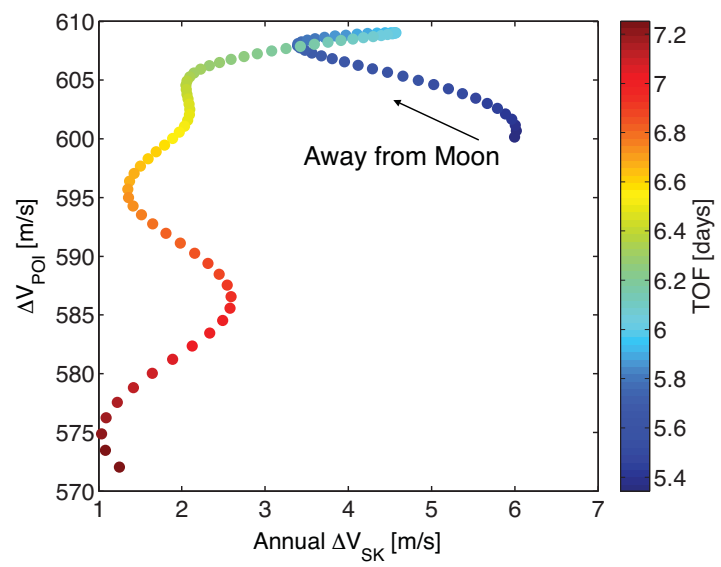

(a) Transfer diagram colored by TOF.

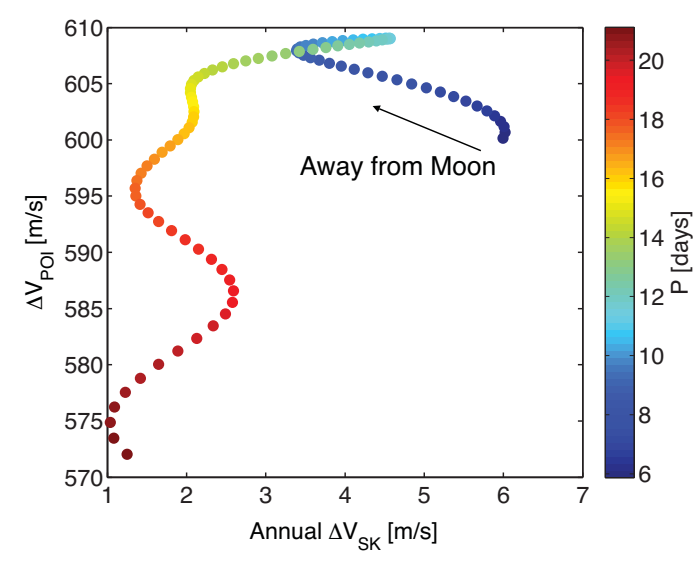

(b) Transfer diagram colored by DRO period.

Figure 12. Diagram of transfer and annual station-keeping costs for orbits sampled along the DRO family.

can be characterized both by low transfer costs and low station-keeping costs. These attributes may be suitable for an infrastructure that is intended to be frequently accessed and maintained.

Upon further examination of the DRO family, a candidate storage orbit can be isolated, one that is accessible, for example, by a crewed vehicle that may also require up to one orbital period for onorbit rendezvous. Recent concepts for manned missions to the lunar vicinity are typically limited to a maximum roundtrip time-of-flight of approximately 21 days, based on restrictions for hardware, supplies, and human factors. ${ }^{13}$ This limit of 21 days includes both the outbound and return legs along a transfer arc, as well as the time-on-orbit. Since the TOF corresponding to a one-way trip to the sample DROs can vary between 5.4 and 7.2 days, a candidate storage orbit must possess an orbital period of, at most, 10 days. This upper bound assumes that on-orbit rendezvous activities may require up to one revolution in the periodic orbit. From Figure 12(b), a user of an interactive database would search for DROs that are represented by blue dots. Assuming that the storage orbit is accessible from either an $L_{1}$ or $L_{2}$ Lyapunov orbit, some sample DROs that satisfy each of these requirements are colored red in Figure 13. The selected candidate storage orbit for an infrastructure asset that is accessible by a human crew is the DRO displayed in Figure 14(a). This orbit possesses a period of 9.39 days, a Jacobi constant equal to 2.97, and requires a flight time of 5.76 days for a direct transfer from LEO. Assuming, at most, one revolution of on-orbit rendezvous activities, this candidate storage orbit meets the requirements for the scenario outlined in this example: the DRO can be maintained over several years for a reasonable station-keeping cost; is accessible from the Earth, as well as members of the $L_{1}$ and $L_{2}$ Lyapunov families; and possesses both a period and geometry that do not place significant limitations on launch opportunities prior to on-orbit rendezvous. Further, Figure 14(b) also depicts the maintenance of this selected DRO over one year using the long term station-keeping strategy employed within this investigation. It is apparent that the selected DRO is well maintained over twelve revolutions, requiring only a few $\mathrm{m} / \mathrm{s}$ in annual station-keeping $\Delta V$. This storage orbit was rapidly and efficiently selected, thereby demonstrating the capabilities of a dynamic reference catalog in an interactive environment. In addition, alternative candidate orbits were also retained throughout this process and could be further examined using a similar analysis. 


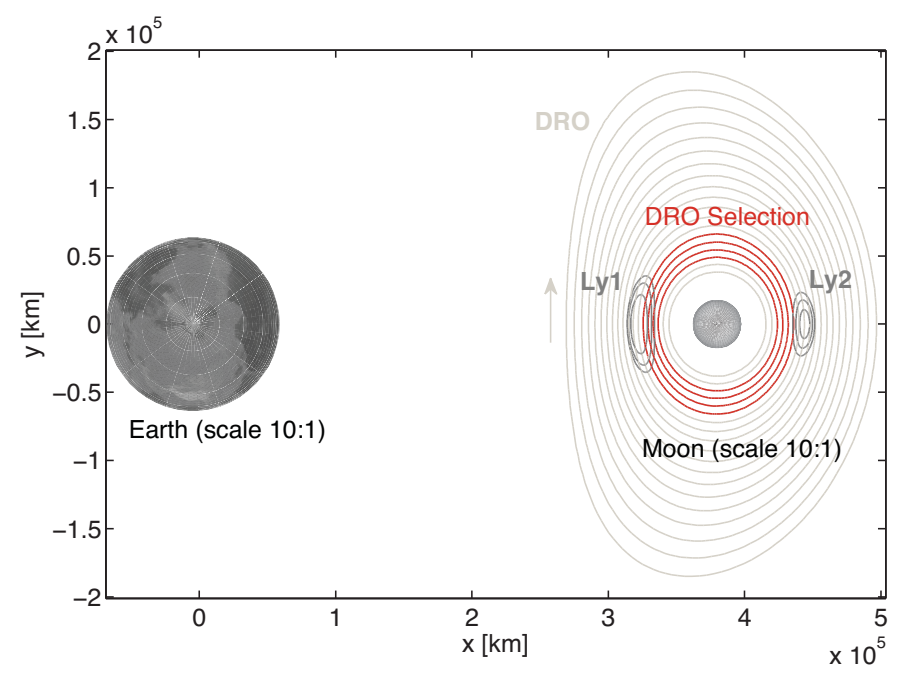

Figure 13. DRO family in the Earth-Moon system with potential storage orbit candidates colored red.

Selected periodic orbits and their corresponding transfers from LEO can also be exported to a trajectory design environment such as ATD, developed at Purdue University, for more complete analysis including the transition to a higher-fidelity model with, potentially, an eventual delivery to GMAT. ${ }^{9}$ As an example, consider a transfer to a DRO with period equal to 6.5 days and Jacobi constant value of 2.91. Given an arbitrary epoch, assumed to be January 1, 2021, the DRO and its corresponding transfer are input to ATD. This interactive trajectory design environment then allows correction of the trajectory using a multiple shooting strategy. An example of a converged transfer from LEO to the given DRO is displayed in Figure 15, and is plotted in an instantaneously defined rotating, barycentered, Earth-Moon frame. Although a precisely periodic orbit does not exist in the ephemeris model, the corrected transfer and DRO approximately retain the same characteristics as the reference solution that was constructed in the CR3BP. Furthermore, the periodic orbit insertion cost for the converged transfer in the ephemeris model does not deviate significantly from the transfer cost estimated in the CR3BP. Accordingly, there is significant merit in using the CR3BP as a simplified model of the Earth-Moon system in the interactive catalog. In addition to the reduction in computational effort and the simplification in visualizing an autonomous system, the CR3BP allows for identification of periodic solutions that predict some of the dynamical structures that are actually present in an ephemeris model of the Earth-Moon space.

\section{CONCLUDING REMARKS}

The concept of an interactive and 'dynamic' catalog of solutions in the Earth-Moon system is explored and demonstrated to offer an environment for rapid and intuitive tradeoffs among orbits that may be exploited during preliminary mission design. This interactive database currently incorporates known periodic solutions in the CR3BP and is focused on comparisons involving libration point orbits, resonant orbits and Moon-centered orbits. The framework is easily expanded as new options evolve. The capability to compute and analyze characteristic parameters of these families 'on demand' via the 'dynamic' catalog allows for the selection of candidate orbits for missions in the Earth-Moon system. These candidate orbits can be exported for further examination in an 
end-to-end trajectory design environment. The capabilities of the interactive reference database are apparent when employed to select a candidate storage orbit for infrastructure that may support lunar activities or enable solar system exploration. In addition, the use of a simplified gravitational model for the Earth-Moon system is verified by noting that the characteristics of orbits that are periodic in the CR3BP are approximately retained when corrected in an ephemeris model.

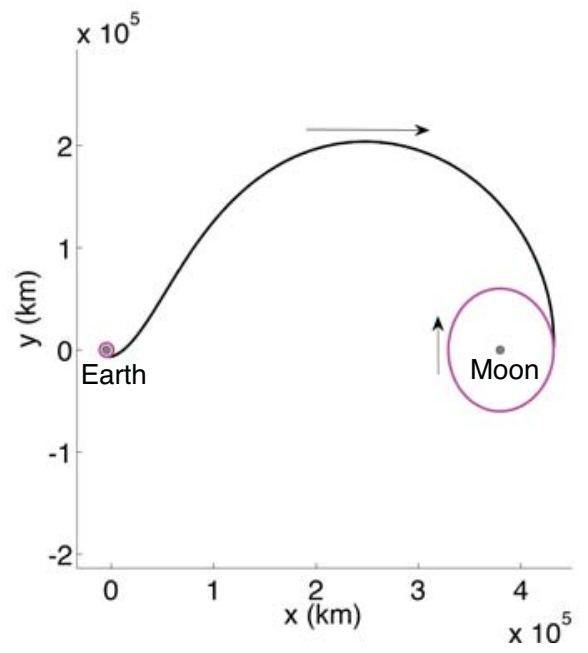

(a) Transfer to DRO from a $300 \mathrm{~km}$ altitude LEO.

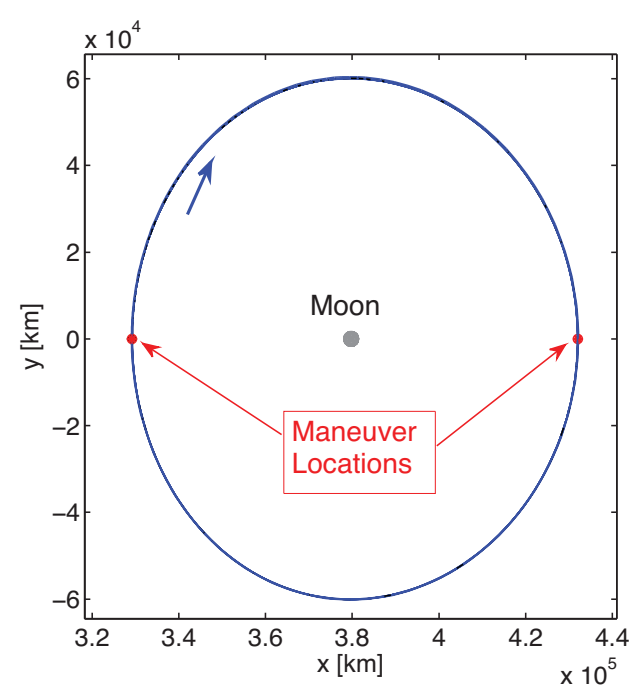

(b) Maintenance of selected DRO using long term station-keeping strategy.

Figure 14. Selected candidate storage orbit, a DRO with period of 9.39 days.

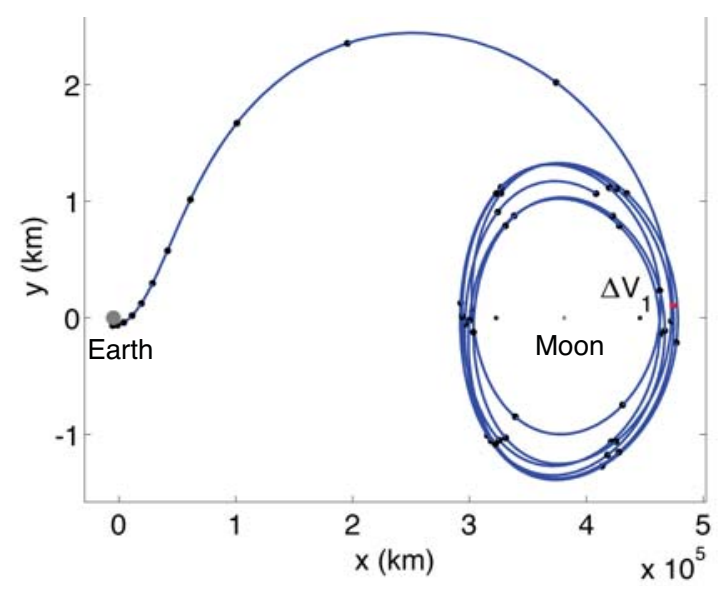

Figure 15. Sample LEO to DRO transfer converged in an ephemeris model for a departure epoch of January 01, 2021 [With permission ${ }^{21}$ ].

\section{ACKNOWLEDGEMENTS}

The authors appreciate the support of the School of Aeronautics and Astronautics. This work was completed at Purdue University under NASA Grants NNX13AM17G and NNX13AH02G. The authors also wish to express their gratitude to Ph.D. candidate Ms. Amanda Haapala and 
Dr. Thomas Pavlak for their valuable insight and assistance with the computational aspects of this research. In addition, many thanks to Dr. Mar Vaquero for early contributions to this work and Ph.D. student Ms. Lucia Capdevila for valuable discussions.

\section{REFERENCES}

[1] B.G. Drake, NASA Decadal Planning Team Mars Mission Analysis Summary, February 2007.

[2] NASA Advanced Development Office, Lunar $L_{1}$ Gateway Conceptual Design Report, October 2001.

[3] NASA Headquarters, Science Mission Directorate, NASA Astrophysics Implementation Plan, December 2012:

[4] International Space Exploration Coordination Group, NASA Global Exploration Roadmap, August 2013.

[5] D.C. Folta, M.A. Woodard, and D. Cosgrove, 2011. "Stationkeeping of the First Earth-Moon Libration Orbiters: The ARTEMIS Mission," AAS/AIAA Astrodynamics Specialist Conference, Alaska. AAS 11515.

[6] Satellite ToolKit, Software Package, Analytical Graphics Inc., Exton, PA, 2012.

[7] General Mission Analysis Tool, Available from gmatcentral.org.

[8] E.J. Doedel, V.A. Romanov, R.C. Paffenroth, H.B. Keller, D.J. Dichmann, J. Galán-Vioque and A. Vanderbauwhede, "Elemental Periodic Orbits Associated with the Libration Points in the Circular Restricted 3-Body Problem". International Journal of Bifurcation and Chaos, Vol 17, No. 8, 2007.

[9] A. Haapala, M. Vaquero, T.A. Pavlak, K.C. Howell, and D. Folta, "Trajectory Selection Strategy for Tours in the Earth-Moon System," AAS/AIAA Astrodynamics Specialist Conference, Hilton Head, South Carolina, August 10-15, 2013.

[10] T.A. Pavlak, "Trajectory Design and Orbit Maintenance Strategies in Multi-body Dynamical Regimes" Ph.D. Dissertation, School of Aeronautics and Astronautics, Purdue University, West Lafayette, Indiana, 2013.

[11] W. S. Koon, M. W. Lo, J. E. Marsden, S. D. Ross, Dynamical Systems, the Three Body Problem and Space Mission Design. Springer-Verlag New York Incorporated, 2011.

[12] L. Perko, Differential Equations and Dynamical Systems. Third Edition, New York, Springer, 2000.

[13] D. Folta, T. Pavlak, A. Haapala, and K.C. Howell, "Preliminary Considerations for Access and Operations in Earth-Moon L1/L2 Orbits," AAS/AIAA Space Flight Mechanics Meeting, Kauai, Hawaii, August 10-14, 2013.

[14] D.G. Yarnoz, J.P. Sanchez and C.R. McInnes, "Pure Opportunities for Asteroid Retrieval Missions," In: Asteroids. Springer Berlin Heidelberg, London, Chapter 21. 2013.

[15] K. Uesugi, "Space Odyssey of an Angel: Summary of the HITEN's Three Year Mission".Advances in the Astronautical Sciences, Vol. 84, pp. 607-621, 1993

[16] J. Carrico Jr., D. Dichmann, L. Policastri, J. Carrico III, T. Craychee, J. Ferreira, M. Intelisano, R. Lebois, M. Loucks, T. Schrift and R. Sherman, "Lunar Resonant Trajectory Design for the Interstellar Boundary Explorer (IBEX) Extended Mission," AAS/AIAA Astrodynamics Specialist Conference, Girdwood, Alaska, July 31-August 4, 2011.

[17] J.W. Gangestad, G.A. Henning, R. Persinger, G.R. Ricker, "A High Earth, Lunar Resonant Orbit for Lower Cost Space Science Missions," AAS/AIAA Space Flight Mechanics Meeting, August 11-15, 2013.

[18] N. Strange, D. Landau, T. McElrath, G. Lantoine, T. Lam, M. McGuire, L. Burke, M. Martini, and J. Dankanich, "Overview of Mission Design for NASA Asteroid Redirect Robotic Mission Concept," International Electric Propulsion Conference, Washington D.C., October 6-10, 2013.

[19] G. Mingotti, F. Topputo, F. Bernelli-Zazzera, "Exploiting Distant Periodic Orbits and their Invariant Manifolds to Design Novel Space Trajectories to the Moon," AAS/AIAA Space Flight Mechanics Meeting, February 14-18, 2010.

[20] M. Kakoi, K.C. Howell, and D. Folta, "Access to Mars from Earth-Moon Libration Point Orbits: Manifold and Direct Options," IAF 64th International Astronautical Congress, Beijing, China, September 23-27, 2013.

[21] L. Capdevila, D. Guzzetti, and K.C. Howell, "Various Transfer Options from Earth into Distant Retrograde Orbits in the Vicinity of the Moon," AAS/AIAA Space Flight Mechanics Meeting, January 26-30, 2014. 